\title{
ON CUBATURE RULES ASSOCIATED TO WEYL GROUP ORBIT FUNCTIONS
}

\author{
Lenka HÁKovÁa, $^{a, *}$, JiŘí HrivnáK ${ }^{b}$, Lenka Motlochová ${ }^{b}$ \\ a Department of Mathematics, Faculty of Chemical Engineering, University of Chemistry and Technology, \\ Prague, Technická 5, CZ-166 28 Prague, Czech Republic \\ ${ }^{b}$ Department of Physics, Faculty of Nuclear Sciences and Physical Engineering, Czech Technical University \\ in Prague, Břehová 7, CZ-115 19 Prague, Czech Republic \\ * corresponding author: lenka.hakova@vscht.cz
}

\begin{abstract}
The aim of this article is to describe several cubature formulas related to the Weyl group orbit functions, i.e. to the special cases of the Jacobi polynomials associated to root systems. The diagram containing the relations among the special functions associated to the Weyl group orbit functions is presented and the link between the Weyl group orbit functions and the Jacobi polynomials is explicitly derived in full generality. The four cubature rules corresponding to these polynomials are summarized for all simple Lie algebras and their properties simultaneously tested on model functions. The Clenshaw-Curtis method is used to obtain additional formulas connected with the simple Lie algebra $C_{2}$.
\end{abstract}

KEYWORDS: Weyl group orbit functions; Jacobi polynomials; cubature formulas.

\section{INTRODUCTION}

The purpose of this paper is to explicitly overview in full generality the link between the Weyl group orbit functions and the Jacobi and Macdonald polynomials and further examine and compare related methods of numerical integration. These methods of numerical integration, known as cubature rules, emerged recently for all four cases of the Weyl group orbit functions.

The four families of the Weyl group orbit functions [10, 18, 19, 26, 30, are connected to four families of orthogonal polynomials via similar relations between Chebyshev polynomials of the first and second kinds and the ordinary cosine and sine functions. A full set of four families of orbit functions, called $C_{-}, S_{-}, S^{s_{-}}$ and $S^{l}$-functions, arises from root systems of simple Lie algebras with two different lengths of roots. These four families of orthogonal polynomials are in fact special cases of the multivariate Jacobi polynomials [11, 12. The Jacobi polynomials associated to root systems are in turn limiting cases of the Macdonald polynomials [24]. This connection between the four cases of the Jacobi polynomials and the underlying orbit functions allows to formulate the corresponding methods for numerical integration in terms of the Jacobi polynomials.

Among methods for numerical integration, the quadrature and cubature formulas related to polynomials of a bounded degree hold a prominent place [1, 68, 35, 38. Such formulas estimate a given weighted integral over a fixed domain in Euclidean space. This estimation holds exactly for all polynomials up to a certain degree. A significant effort put into development of various types of cubature formulas results in multitude of types of integration domains with varying efficiencies. The shapes of the integration domains and the nodes for cubature formulas corresponding to the orthogonal polynomials of the Weyl group orbit functions are determined by the symmetries of the affine Weyl groups and a certain transform [15, 22, 23, 26, 27. This transform is generated by the transform which induces the given set of orthogonal polynomials. Moreover, a specific notion of the modified degree of multivariate polynomials is essential for establishing the final cubature formulas.

One of the specific methods of deriving quadrature formulas, known as Clenshaw-Curtis method [5], is classically related to Chebyshev polynomials of one variable 9 . Its two-dimensional version related to twovariable Chebyshev polynomials of the root system $A_{2}$ is also developed 31. The importance of this method lies e.g. in its utilization for practical optimization of the shapes of integration domains. The shapes of the integration domains are determined by the underlying Lie algebra [15] and are, however, of non-standard form. In case of simple Lie algebras related to twovariable functions, one of the possible optimizations of these shapes is, similarly to [31, inscribing a triangle into the original fundamental domain. The focus of the present article is on simple Lie algebra $C_{2}$ and its corresponding cubature rules.

The integration domain in the case of $C_{2}$ is a region bounded by two lines and a parabola depicted in Fig. 4. Except from a general perspective in [15, 26, 27, integration over this region is studied in [32]. Similarly to [31] for $A_{2}$, the non-standard shape of this integration domain motivates further exploration of the ClenshawCurtis method. This method crucially depends on the choice of the weight function and the inscribed integration region and has not yet been studied in detail for 
the case of $C_{2}$. In this case, the domain inscribed in the original integration region is considered either the original domain itself or the triangle depicted in Fig. 6 Two fundamental choices of the weight functions are detailed. Prior to practical implementation, exact values of certain integrals are also needed. One of the goals of this article is to provide all data necessary for practical implementation of these cubature formulas. This is achieved by tabulating and calculating all needed stabilizer coefficients and exact integral values for each choice it the inscribed integration domain and weight function. To demonstrate usefulness and viability of the presented methods, the numerical tests on model functions, including multidimensional step-functions, are also performed.

The development of novel cubature formulas is motivated by their widespread use in applied numerical simulations and engineering problems. Among direct numerical applications of the cubature formulas is the induced method of polynomial approximation. The cubature formulas are ubiquitous in the modern theory of electromagnetism, especially in its branches of electromagnetic wave propagation [33], magnetostatic modeling [39] and micromagnetic simulations [4]. Other fields include fluid flows simulations, laser optics and stochastic dynamics.

In Section 2 are reviewed the notions necessary for definition of the Weyl group orbit functions. The relation between the orbit functions and the Jacobi polynomials is detailed. In Section 3 , the cubature formulas from [15, 26, 27] are summarized, ClenshawCurtis method is described and used to derive additional cubature formulas. Furthermore, numerical test results are presented.

\section{SPECIAL FUNCTIONS ASSOCIATED TO ROOT SYSTEMS}

\subsection{BASIC DEFINITIONS}

This section reviews the basic concepts and notation from the theory of root systems, Weyl groups and Weyl group orbit functions. It is consistent with the notation used in recent papers regarding the topic, such as 10, 13, 15, 26] and others. We consider simple Lie algebras, i.e. four infinite families $A_{n}(n \geq 1)$, $B_{n}(n \geq 3), C_{n}(n \geq 2)$ and $D_{n}(n \geq 4)$ and five exceptional algebras $E_{6}, E_{7}, E_{8}, F_{4}$ and $G_{2}$ (for the classification see [2, 16]). In particular, we focus on the algebra $C_{2}$ as the simplest non-trivial example. Each simple Lie algebra is completely described by its set of simple roots $\Delta=\left\{\alpha_{1}, \ldots, \alpha_{n}\right\}$ which forms a nonorthogonal basis of the Euclidean space $\mathbb{R}^{n}$ equipped with a scalar product denoted by $\langle\cdot, \cdot\rangle$. Simple roots are either of the same length or of two different lengths, in the latter case we distinguish so-called short and long roots and write $\Delta=\Delta_{s} \cup \Delta_{l}$.

The set of dual roots is denoted by

$$
\Delta^{\vee}=\left\{\alpha_{1}^{\vee}, \ldots, \alpha_{n}^{\vee}\right\}
$$

where $\alpha_{i}^{\vee}=\frac{2 \alpha_{i}}{\left\langle\alpha_{i}, \alpha_{i}\right\rangle}$. In addition to the bases of simple roots and dual roots we introduce the weight basis $\omega_{1}, \ldots, \omega_{n}$ and the dual weight basis $\omega_{1}^{\vee}, \ldots, \omega_{n}^{\vee}$ where $\left\langle\alpha_{i}^{\vee}, \omega_{j}\right\rangle=\left\langle\alpha_{i}, \omega_{j}^{\vee}\right\rangle=\delta_{i j}$. The Cartan matrix $C$ is defined as $C_{i j}=\frac{2\left\langle\alpha_{i}, \alpha_{j}\right\rangle}{\left\langle\alpha_{j}, \alpha_{j}\right\rangle}$ and its determinant is denoted by $c$.

Each simple root $\alpha_{i}$ relates to a reflection $r_{i}$ defined for every $a \in \mathbb{R}^{n}$ as

$$
r_{i} a=a-\frac{2\left\langle a, \alpha_{i}\right\rangle}{\left\langle\alpha_{i}, \alpha_{i}\right\rangle} \alpha_{i}
$$

The set of reflections $\left\{r_{1}, \ldots, r_{n}\right\}$ generates a finite group $W$ called the Weyl group. By the action of $W$ on the set of simple roots we obtain the root system $\Pi=$ $W \Delta$. Analogously, we define $\Pi^{\vee}=W \Delta^{\vee}, \Pi^{s}=W \Delta_{s}$ and $\Pi^{l}=W \Delta_{l}$. Every element of $\Pi$ can be written as a combination of simple roots with only non-negative (positive roots) or non-positive integer coefficients (negative roots). The set of positive roots is denoted by $\Pi_{+}$. We define a partial ordering $\preceq$ of roots, $\mu \preceq \lambda$ if $\mu-\lambda$ is a sum of simple roots with non-negative integer coefficients. There is a unique highest root $\xi$ with respect to this ordering, its coordinates in the basis of simple roots are called marks and denoted by $m_{1}, \ldots, m_{n}$. Dual root system $\Pi^{\vee}$ contains the highest root $\eta=m_{1}^{\vee} \alpha_{1}^{\vee}+\cdots+m_{n}^{\vee} \alpha_{n}^{\vee}$ with the coefficients $m_{i}^{\vee}$ called dual marks.

An infinite extension of the Weyl group $W$ is the affine Weyl group $W^{\text {aff }}$ which is obtained by adding to the set of generators of $W$ the affine reflection $r_{0}$,

$$
r_{0} a=r_{\xi} a+\frac{2 \xi}{\langle\xi, \xi\rangle}, \quad r_{\xi} a=a-\frac{2\langle a, \xi\rangle}{\langle\xi, \xi\rangle} \xi .
$$

It can also be written as a semidirect product of $W$ and a set of shifts by integer combinations of dual roots [13. We denote by $\psi$ the retraction homomorphism $W^{\text {aff }} \rightarrow$ $W$ 14. The fundamental domain $F$ - a set containing exactly one point from each $W^{\text {aff }}$ orbit - can be chosen as

$$
\begin{aligned}
F= & \left\{b_{1} \omega_{1}^{\vee}+\cdots+b_{n} \omega_{n}^{\vee} \mid\right. \\
& \left.b_{i} \in \mathbb{R}^{\geq 0}, b_{0}+b_{1} m_{1}+\cdots+b_{n} m_{n}=1\right\} .
\end{aligned}
$$

Analogously we define dual affine Weyl group as a semidirect product of $W$ and shifts by integer combinations of simple roots.

We introduce three lattices $P, P^{+}$and $P^{\vee}$ as

$$
\begin{aligned}
P & =\mathbb{Z} \omega_{1}+\cdots+\mathbb{Z} \omega_{n}, \\
P^{+} & =\mathbb{Z}^{\geq 0} \omega_{1}+\cdots+\mathbb{Z}^{\geq 0} \omega_{n}, \\
P^{\vee} & =\mathbb{Z} \omega_{1}^{\vee}+\cdots+\mathbb{Z}_{n}^{\vee} .
\end{aligned}
$$

Note that the root system $\Pi$ is contained in $P$, therefore, the partial ordering $\preceq$ can be extended to the lattice $P$.

A function $k: \alpha \in \Pi \rightarrow k_{\alpha} \in \mathbb{R}^{\geq 0}$ such that

$$
k_{\alpha}=k_{w(\alpha)} \quad \text { for all } \quad w \in W
$$


is known as a multiplicity function on $\Pi$. The trivial example is to take $k_{\alpha}=$ const for all $\alpha \in \Pi$ which we denote by $k^{\text {const }}$. For simple Lie algebras with two different root lengths, it is natural to distinguish between short and long roots by defining

$$
k_{\alpha}^{s} \equiv\left\{\begin{array} { l l } 
{ 1 } & { \text { if } \alpha \in \Pi ^ { s } , } \\
{ 0 } & { \text { if } \alpha \in \Pi ^ { l } , }
\end{array} \quad k _ { \alpha } ^ { l } \equiv \left\{\begin{array}{ll}
0 & \text { if } \alpha \in \Pi^{s} \\
1 & \text { if } \alpha \in \Pi^{l} .
\end{array}\right.\right.
$$

The notion of multiplicity function allows us to define sums of positive roots $\varrho(k)$ and numbers $h(k)$,

$$
\begin{aligned}
& \varrho(k) \equiv \frac{1}{2} \sum_{\alpha \in \Pi_{+}} k_{\alpha} \alpha, \\
& h(k)=k_{\xi}+\sum_{i=1}^{n} m_{i} k_{\alpha_{i}} .
\end{aligned}
$$

In particular, with the choice of $k^{t}$, where $t$ is one of the symbols $\{0,1, s, l\}$, we have

$$
\begin{aligned}
& \varrho^{0} \equiv \varrho\left(k^{0}\right)=0 \\
& \varrho^{1} \equiv \varrho\left(k^{1}\right)=\sum_{i=1}^{n} \omega_{i}, \\
& \varrho^{s} \equiv \varrho\left(k^{s}\right)=\sum_{\alpha_{i} \in \Delta_{s}} \omega_{i}, \\
& \varrho^{l} \equiv \varrho\left(k^{l}\right)=\sum_{\alpha_{i} \in \Delta_{l}} \omega_{i}
\end{aligned}
$$

and

$$
\begin{aligned}
& h^{0} \equiv h\left(k^{0}\right)=0, \\
& h^{1} \equiv h\left(k^{1}\right)=1+\sum_{i=1}^{n} m_{i}, \\
& h^{s} \equiv h\left(k^{s}\right)=\sum_{\alpha_{i} \in \Delta_{s}} m_{i}, \\
& h^{l} \equiv h\left(k^{l}\right)=1+\sum_{\alpha_{i} \in \Delta_{l}} m_{i} .
\end{aligned}
$$

The number $h \equiv h^{1}$ is called the Coxeter number, analogously, we call $h^{s}$ and $h^{l}$ short and long Coxeter number.

The set of simple roots $\Delta=\left\{\alpha_{1}, \alpha_{2}\right\}$ of the algebra $C_{2}$ decomposes into the set of the short simple roots $\Delta_{s}=\left\{\alpha_{1}\right\}$ and the set of the long simple roots $\Delta_{l}=$ $\left\{\alpha_{2}\right\}$. The highest root is of the form $\xi=2 \alpha_{1}+\alpha_{2}$ and the dual highest root is $\eta=\alpha_{1}^{\vee}+2 \alpha_{2}^{\vee}$. Thus, the sets of mark and dual marks are $\left(m_{1}, m_{2}\right)=(2,1)$ and $\left(m_{1}^{\vee}, m_{2}^{\vee}\right)=(1,2)$. The vectors $\varrho^{t}$ are of the form $\left(\varrho^{1}, \varrho^{s}, \varrho^{l}\right)=\left(\omega_{1}+\omega_{2}, \omega_{1}, \omega_{2}\right)$ and the Coxeter numbers are $\left(h^{1}, h^{s}, h^{l}\right)=(4,2,2)$. The roots and dual roots, the weights and dual weights, together with the fundamental domain $F$ and the vectors $\varrho^{t}$ are depicted in Figure 1

\subsection{WEYL GROUP ORBIT FUNCTIONS}

The definition of Weyl group orbit function uses the notion of a sign homomorphisms $\sigma^{t}: W \mapsto \pm 1$, where

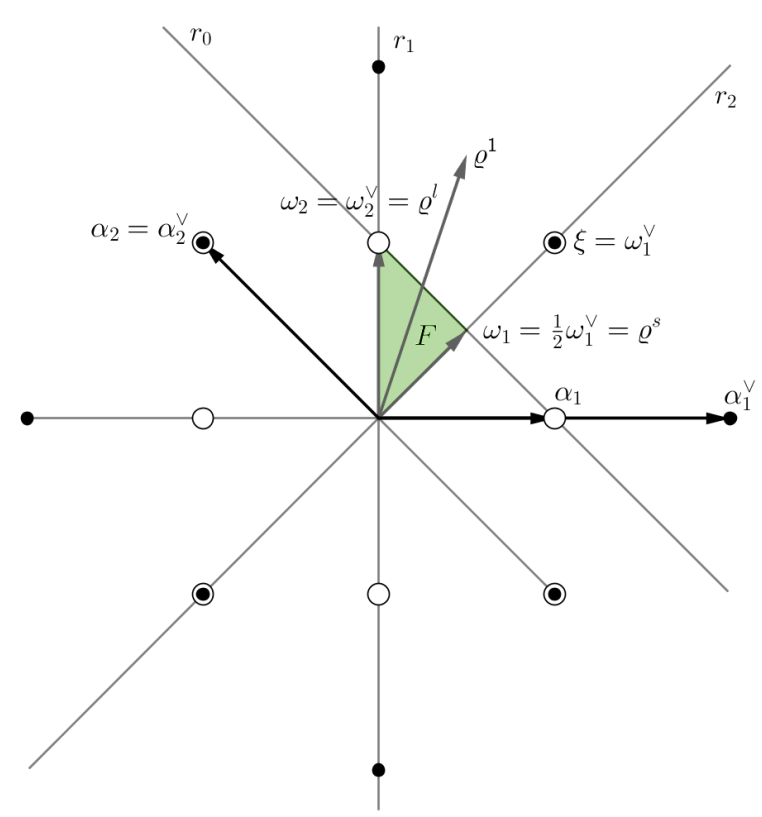

Figure 1. Root system of $C_{2}$. The white circles denote the roots, black dots depict the dual roots. The triangle denotes the fundamental domain $F$. The lines denoted $r_{1}, r_{2}$ and $r_{0}$ depict reflecting mirrors which realize the corresponding reflections.

$t \in\{0,1, s, l\}$. These can be defined by the values on the reflections corresponding to the simple roots $r_{i}$, namely

$$
\begin{aligned}
\sigma^{0}\left(r_{i}\right) & =1, \quad \alpha_{i} \in \Delta, \\
\sigma^{1}\left(r_{i}\right) & =-1, \quad \alpha_{i} \in \Delta, \\
\sigma^{s}\left(r_{i}\right) & = \begin{cases}1 & \text { if } \alpha_{i} \in \Delta_{l}, \\
-1 & \text { if } \alpha_{i} \in \Delta_{s},\end{cases} \\
\sigma^{l}\left(r_{i}\right) & = \begin{cases}1 & \text { if } \alpha_{i} \in \Delta_{s}, \\
-1 & \text { if } \alpha_{i} \in \Delta_{l} .\end{cases}
\end{aligned}
$$

Several families of special functions are connected with each Weyl group $W$. They are labelled by vectors $\lambda \in P^{+}$and defined as weighed sums over the corresponding Weyl group orbit, i.e. the set $W \lambda=\{w \lambda \mid w \in W\}$. For every $x \in \mathbb{R}^{n}$ and $t \in\{0,1, s, l\}$ we define

$$
S_{\lambda+\varrho^{t}}^{t}(x)=\sum_{\mu \in W\left(\lambda+\varrho^{t}\right)} \sigma^{t}(\mu) e^{2 \pi i\langle\mu, x\rangle},
$$

where $\varrho^{t}$ is given by (3) and $\sigma^{t}(\mu) \equiv \sigma^{t}(w)$ for $w$ such that $\mu=w\left(\lambda+\varrho^{t}\right)$. Functions corresponding to the choice of $t=0$ and $t=1$ are usually called $C$ - and $S$ functions respectively, in the formulas in next sections we use the notation $S^{0}$ and $S^{1}$ for a simplicity.

Families of $C_{-}, S_{-}, S^{s_{-}}$and $S^{l}$-functions are complex multivariate functions with remarkable properties such as (anti-)invariance with respect to the action of the affine Weyl group, continuous and discrete orthogonality. They were studied in many papers, see for example [18, 19, 26] for the general properties and [13, 14, 28, for their discretization. 
Fundamental domains $F^{t}$ are defined as subsets of $F$ such that we omit the part of the boundary of $F$ which is stabilized by certain generating reflections $r \in R=\left\{r_{0}, r_{1} \ldots, r_{n}\right\}$. More precisely, with the notation

$$
\begin{aligned}
& R^{t}=\left\{r \in R \mid \sigma^{t} \circ \psi(r)=-1\right\}, \\
& H^{t}=\left\{a \in F \mid \exists r \in R^{t}, r a=a\right\},
\end{aligned}
$$

we define $F^{t}=F \backslash H^{t}$. The explicit forms are obtained from (1) and can be found in [14].

The $C$-, $S$-, $S^{s}$ - and $S^{l}$-functions can be viewed as functional forms of elements from the algebra $\mathbb{C}[P]$ containing all complex linear combinations of formal exponentials $e^{a}, a \in P$, with multiplication defined by $e^{a} \cdot e^{b}=e^{a+b}$, the inverse given by $\left(e^{a}\right)^{-1}=e^{-a}$ and the identity $e^{0}=1$. The connection is based on the exponential mapping from Lie algebra to the corresponding Lie group [2, 16, 27].

\subsection{JACOBI POLYNOMIALS}

We assume that the multiplicity function $k$ satisfies $k_{\alpha} \geq 0$. The Jacobi polynomial $P(\lambda, k)$ [11, 12 associated to the root system $\Pi$ with highest weight $\lambda \in P^{+}$ and multiplicity function $k$ as parameter is defined by the following formulas.

$$
P(\lambda, k) \equiv \sum_{\substack{\mu \in P^{+} \\ \mu \preceq \lambda}} c_{\lambda \mu}(k) C_{\mu}, \quad C_{\mu}=\sum_{\mu^{\prime} \in W \mu} e^{\mu^{\prime}},
$$

where the coefficients $c_{\lambda \mu}(k)$ are recursively given by

$$
\begin{array}{r}
(\langle\lambda+\varrho(k), \lambda+\varrho(k)\rangle-\langle\mu+\varrho(k), \mu+\varrho(k)\rangle) c_{\lambda \mu}(k) \\
=2 \sum_{\alpha \in \Pi_{+}} k_{\alpha} \sum_{j=1}^{\infty}\langle\mu+j \alpha, \alpha\rangle c_{\lambda, \mu+j \alpha}(k)
\end{array}
$$

along with the initial value $c_{\lambda \lambda}=1$ and the assumption $c_{\lambda \mu}=c_{\lambda, w(\mu)}$ for all $w \in W$. Recall that $\varrho(k)$ is defined by (2).

By setting $k_{\alpha}=0$ for all $\alpha \in \Pi$, the Jacobi polynomials lead trivially to $C$-functions. In the case $k=k^{1}$, the formula for the calculation of the coefficients becomes the Freudenthal's recurrence formula [16]. Therefore, each $P\left(\lambda, k^{1}\right)$ specializes to a character $\chi_{\lambda}$ of an irreducible representation of the simple Lie algebra of the highest weight $\lambda$, i.e.,

$$
P\left(\lambda, k^{1}\right)=\chi_{\lambda}=\frac{S_{\lambda+\varrho^{1}}}{S_{\varrho^{1}}} .
$$

In addition, we show that the Jacobi polynomials are related to the $S^{s}$ - and $S^{l}$-functions in the following way.

$$
P\left(\lambda, k^{s}\right)=\frac{S_{\lambda+\varrho^{s}}^{s}}{S_{\varrho^{s}}^{s}} \quad \text { and } \quad P\left(\lambda, k^{l}\right)=\frac{S_{\lambda+\varrho^{l}}^{l}}{S_{\varrho^{l}}^{l}} .
$$

We first observe that $S_{\lambda+\varrho^{s}}^{s} / S_{\varrho^{s}}^{s}$ are Weyl group invariant elements of $\mathbb{C}[P]$ (see Proposition 4.2 of [26]) with well-known basis formed by $C$-functions [2]. Therefore, each $S_{\lambda+\rho^{s}}^{s} / S_{\varrho^{s}}^{s}$ can be expressed as a linear combination of $C$-functions. By the definition of the Weyl group, the weights of exponentials in $S_{\lambda+\varrho^{s}}^{s}$ are of the form $\lambda+\varrho^{s}-g\left(\alpha_{1}, \ldots, \alpha_{n}\right)$, where $g\left(\alpha_{1}, \ldots, \alpha_{n}\right)$ denotes a sum of simple roots with non-negative integer coefficients, and the unique maximal weight is $\lambda+\varrho^{s}$. Similarly, the unique maximal weight of $S_{\varrho^{s}}^{s}$ is $\varrho^{s}$. Therefore, we have

$$
\frac{S_{\lambda+\varrho^{s}}^{s}}{S_{\varrho^{s}}^{s}}=\sum_{\substack{\mu \in P^{+} \\ \mu \preceq \lambda}} b_{\mu} C_{\mu}, \quad b_{\lambda}=1 .
$$

To prove $b_{\mu}=c_{\lambda \mu}\left(k^{s}\right)$, we proceed by using an equivalent definition of Jacobi polynomials with the multiplicity function satisfying $k_{\alpha} \in \mathbb{Z}^{\geq 0}[12$. For any $f=\sum_{\lambda} a_{\lambda} e^{\lambda}$, we define

$$
\bar{f} \equiv \sum_{\lambda} \bar{a}_{\lambda} e^{-\lambda} \quad \text { and } \quad C T(f) \equiv a_{0} .
$$

If we introduce the scalar product $(\cdot, \cdot)$ on $\mathbb{C}[P]$ by

$$
\begin{gathered}
(f, g) \equiv C T\left(f \bar{g} \delta(k)^{\frac{1}{2}} \overline{\delta(k)^{\frac{1}{2}}}\right), \quad f, g \in \mathbb{C}[P], \\
\delta(k)^{\frac{1}{2}} \equiv \prod_{\alpha \in \Pi_{+}}\left(e^{\frac{1}{2} \alpha}-e^{-\frac{1}{2} \alpha}\right)^{k_{\alpha}}
\end{gathered}
$$

then the Jacobi polynomials $P(\lambda, k)$ are the unique polynomials of the form (6) satisfying the requirement

$$
(P(\lambda, k), P(\mu, k))=0
$$

for all $\mu \in P^{+}$such that $\mu \preceq \lambda$ and $\lambda \neq \mu$ assuming $c_{\lambda \lambda}=1$.

Using Proposition 4.1 of [26], i.e. $\delta\left(k^{s}\right)^{\frac{1}{2}}=S_{\varrho^{s}}^{s}$, we obtain

$$
\begin{aligned}
& \left(\frac{S_{\lambda+\varrho^{s}}^{s}}{S_{\varrho^{s}}^{s}}, \frac{S_{\mu+\varrho^{s}}^{s}}{S_{\varrho^{s}}^{s}}\right)=C T\left(S_{\lambda+\varrho^{s}}^{s} \overline{S_{\mu+\varrho^{s}}^{s}}\right) \\
= & C T\left(\sum_{\lambda^{\prime} \in W\left(\lambda+\varrho^{s}\right)} \sum_{\mu^{\prime} \in W\left(\mu+\varrho^{s}\right)} \sigma^{s}\left(\lambda^{\prime}\right) \sigma^{s}\left(\mu^{\prime}\right) e^{\lambda^{\prime}-\mu^{\prime}}\right) .
\end{aligned}
$$

Clearly $\lambda^{\prime}=\mu^{\prime}$ if and only if there exists $w \in W$ such that $\lambda+\varrho^{s}=w\left(\mu+\varrho^{s}\right)$. For we consider $\lambda \in P^{+}$ different from $\mu \in P^{+}$, it is not possible to have $\lambda^{\prime}=\mu^{\prime}$. This implies that

$$
\left(\frac{S_{\lambda+\varrho^{s}}^{s}}{S_{\varrho^{s}}^{s}}, \frac{S_{\mu+\varrho^{s}}^{s}}{S_{\varrho^{s}}^{s}}\right)=0 \text { and } P\left(\lambda, k^{s}\right)=\frac{S_{\lambda+\varrho^{s}}^{s}}{S_{\varrho^{s}}^{s}} .
$$

The proof for the long root case is similar.

Finally, note that the Jacobi polynomials can be viewed as the limiting case of the Macdonald polynomials $P_{\lambda}\left(q, t_{\alpha}\right)$ when $t_{\alpha}=q^{k_{\alpha}}$ with $k_{\alpha}$ fixed and $q \rightarrow 1$. See [24] for more details. The relations among several special functions associated with the Weyl groups, which are summarized in 29, are depicted in Figure 2 


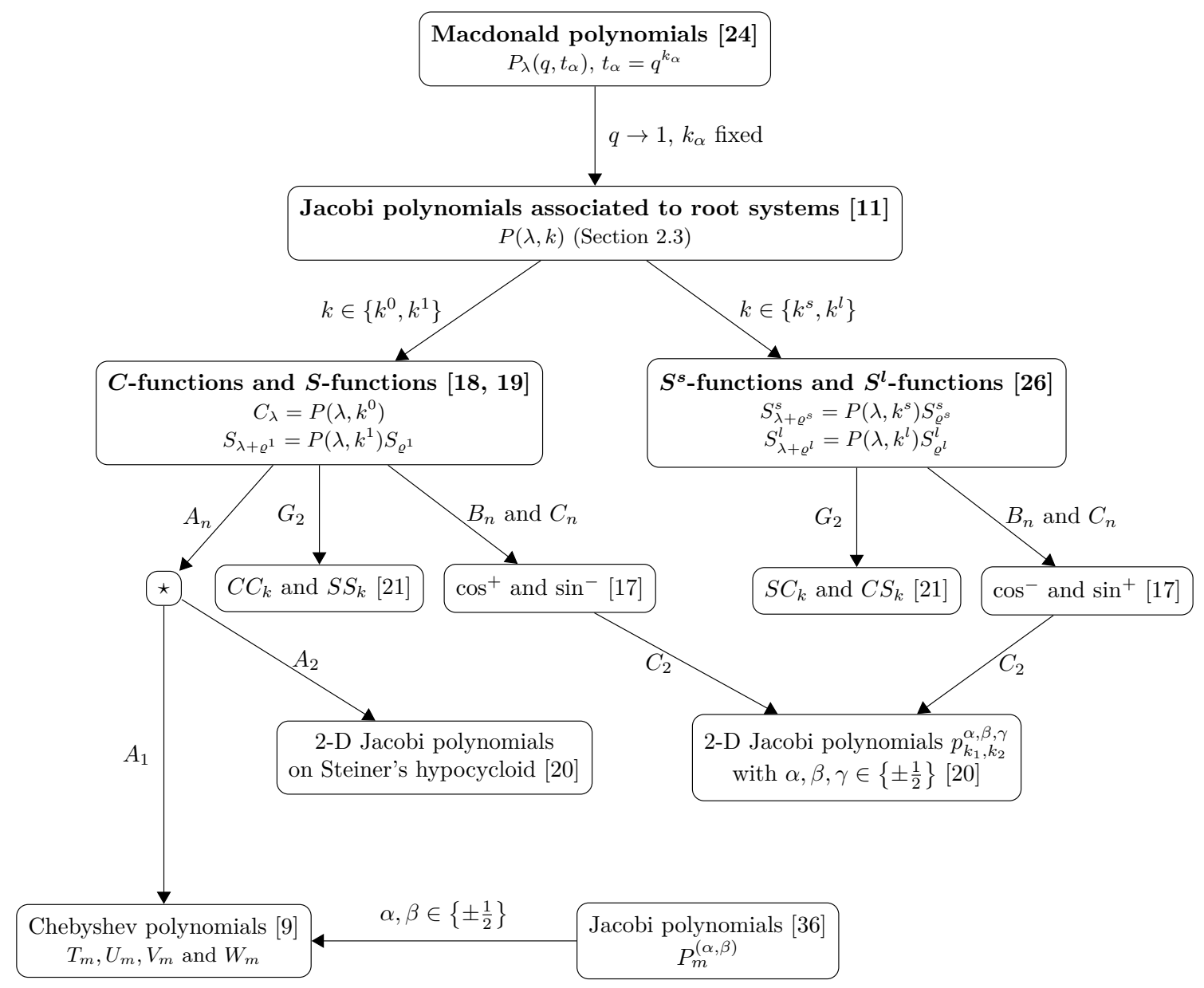

FIgURE 2. The diagram of relations among several special functions associated with Weyl groups.

\section{Cubature formulas}

\subsection{General Form of Cubature formulas}

Analogously to Chebyshev polynomials, we identify polynomial variables $y_{1}, \ldots, y_{n}$ with real-valued functions in the following way. Let $z_{j} \equiv C_{\omega_{j}}$, then

$$
\begin{array}{r}
A_{2 k}: y_{j}=\Re\left(z_{j}\right), y_{2 k-j+1}=\Im\left(z_{j}\right), j=1, \ldots, k, \\
A_{2 k+1}: y_{j}=\Re\left(z_{j}\right), y_{k+1}=z_{k+1}, y_{2 k-j+2}=\Im\left(z_{j}\right), \\
j=1, \ldots, k, \\
D_{2 k+1}: y_{j}=z_{j}, y_{2 k}=\Re\left(z_{2 k}\right), \\
y_{2 k+1}=\Im\left(z_{2 k}\right), j=1, \ldots, 2 k-1, \\
E_{6}: y_{1}=\Re\left(z_{1}\right), y_{2}=\Re\left(z_{2}\right), y_{3}=z_{3}, \\
y_{4}=\Im\left(z_{2}\right), y_{5}=\Im\left(z_{1}\right), y_{6}=z_{6},
\end{array}
$$

otherwise we put $y_{j}=z_{j}$. We say that a monomial $y_{1}^{\lambda_{1}} \ldots y_{n}^{\lambda_{n}}$ has an $m$-degree

$$
\operatorname{deg}_{m} y_{1}^{\lambda_{1}} \ldots y_{n}^{\lambda_{n}}=m_{1}^{\vee} \lambda_{1}+\cdots+m_{n}^{\vee} \lambda_{n}
$$

and any polynomial $p$ in $\mathbb{C}\left[y_{1}, \ldots, y_{n}\right]$ has an $m$-degree equal to the largest $m$-degree of the monomials occurring in $p$. We denote the subspace containing all polynomials of $m$-degree at most $M$ by $\Pi_{M}$. For $\lambda=\lambda_{1} \omega_{1}+\cdots+\lambda_{n} \omega_{n}$, the $C$-functions $C_{\lambda}$ and thus all Jacobi polynomials $P(\lambda, k)$ can be rewritten as orthogonal polynomials in variables $y_{1}, \ldots, y_{n}$ of $m$ degree equal to $m_{1}^{\vee} \lambda_{1}+\cdots+m_{n}^{\vee} \lambda_{n}$ [15].

The variables $y_{1}, \ldots, y_{n}$ viewed as functions induce a map

$$
\Xi: \mathbb{R}^{n} \rightarrow \mathbb{R}^{n}, \quad \Xi(x)=\left(y_{1}(x), \ldots, y_{n}(x)\right) .
$$

The map $\Xi$ is used to define the integration region $\Omega$ and the sets of nodes $\Omega_{M}^{t}, t \in\{0,1, s, l\}$ by

$$
\Omega \equiv \Xi\left(F^{1}\right), \quad \Omega_{M}^{t} \equiv \Xi\left(\frac{1}{M+h^{t}} P^{\vee} \cap F^{t}\right) .
$$

Let

$$
\begin{aligned}
\operatorname{Stab}_{W^{\mathrm{aff}}}(x) & \equiv\left\{w \in W^{\mathrm{aff}} \mid w x=x\right\}, \\
\varepsilon(x) & \equiv \frac{|W|}{\left|\operatorname{Stab}_{W^{\mathrm{aff}}}(x)\right|},
\end{aligned}
$$

and define a map $\widetilde{\varepsilon}: \Omega_{M}^{0} \rightarrow \mathbb{N}$ by

$$
\widetilde{\varepsilon}(y) \equiv \varepsilon\left(\Xi_{M}^{-1} y\right), \quad \Xi_{M}=\Xi \uparrow_{\frac{1}{M+h^{t}}} P^{\vee} \cap F^{0} .
$$

Denoting $K\left(y_{1}, \ldots, y_{n}\right) \equiv \sqrt{S_{\varrho^{1}} \overline{S_{\varrho^{1}}}}$, the weight functions are given by

$$
\begin{aligned}
s^{t}\left(y_{1}, \ldots, y_{n}\right) & \equiv S_{\varrho^{t}}^{t} \overline{S_{\varrho^{t}}^{t}}, \\
w^{t}\left(y_{1}, \ldots, y_{n}\right) & \equiv \frac{s^{t}\left(y_{1}, \ldots, y_{n}\right)}{K\left(y_{1}, \ldots, y_{n}\right)}, \quad t \in\{0,1, s, l\} .
\end{aligned}
$$




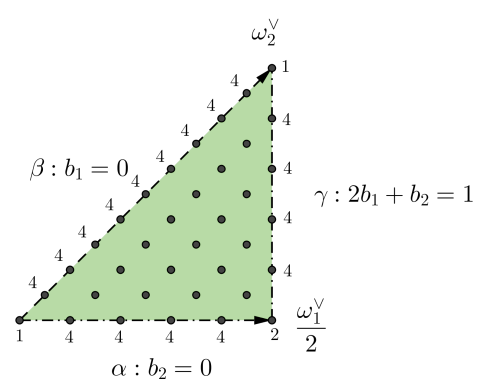

Figure 3 . The fundamental domain $F=F^{0}$ of $C_{2}$ is depicted as the triangle with dashed boundary $H^{s}$ and dot-and-dashed boundary $H^{l}$. The black dots correspond to the points from $\frac{1}{10} P^{\vee} \cap F$. The numbers $1,2,4$ of the dots are the values of $\varepsilon(x)$, the inner dots have $\varepsilon(x)=8$.

Since the products $S_{\varrho^{t}}^{t} \overline{S_{\varrho^{t}}^{t}}, t \in\{0,1, s, l\}$ are $W$ invariant sums of exponentials from $\mathbb{C}[P]$, they are expressible as functions in polynomial variables $y_{1}, \ldots, y_{n}$.

In [15, 26, 27] is shown that the following cubature formulas are exact equalities for any $M \in \mathbb{N}$ and any polynomial $p$ which satisfies the following constraints,

- $\operatorname{deg}_{m} p \leq 2 M-1$ for $t \in\{0, l\}$,

- $\operatorname{deg}_{m} p \leq 2 M+1$ for $t \in\{1, s\}$.

Thus, it holds that

$$
\begin{aligned}
& \int_{\Omega} p(y) w^{t}(y) d y \\
& =\frac{\kappa}{c|W|}\left(\frac{2 \pi}{M+h^{t}}\right)^{n} \sum_{y \in \Omega_{M}^{t}} \widetilde{\varepsilon}(y) s^{t}(y) p(y),
\end{aligned}
$$

where

$$
\kappa= \begin{cases}2^{-\left\lfloor\frac{n}{2}\right\rfloor} & \text { for } A_{n} \\ \frac{1}{2} & \text { for } D_{2 k+1} \\ \frac{1}{4} & \text { for } E_{6} \\ 1 & \text { otherwise }\end{cases}
$$

To numerically compare the efficiency of these cubature formulas, we may consider an integrable function $f$, such that $f / w^{t}$ is well defined on $\Omega_{M}^{t}$, and rewrite the cubatures (7) in the following form:

$$
\begin{aligned}
I(f) & \equiv \int_{\Omega} f(y) d y \approx I_{M}^{t}(f), \\
I_{M}^{t}(f) & \equiv \frac{\kappa}{c|W|}\left(\frac{2 \pi}{M+h^{t}}\right)^{n} \sum_{y \in \Omega_{M}^{t}} \widetilde{\varepsilon}(y) K(y) f(y) .
\end{aligned}
$$

\subsection{Cubature formulas of $C_{2}$}

In this section, the general cubature formulas (8) are specialized and tested on model examples for the case of algebra $C_{2}$. The region $F^{t}$ of $C_{2}$ with the points from $\frac{1}{10} P^{\vee} \cap F^{t}$ is depicted in Fig. 3, whereas the corresponding integration region $\Omega$ with the transformed grid points is depicted in Fig. 4. Note that

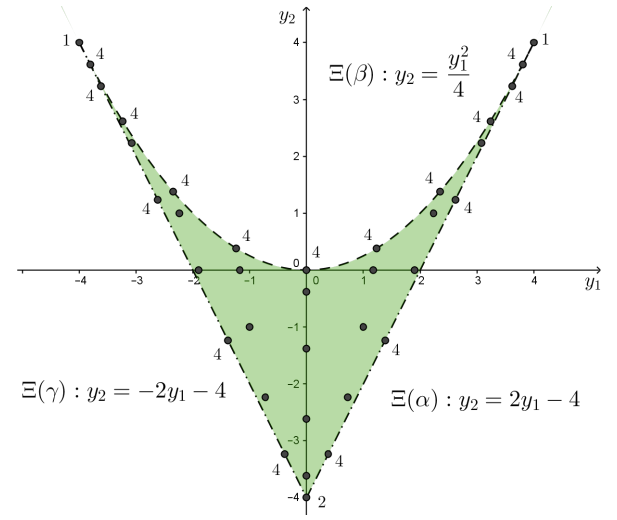

FiguRE 4 . The integration region $\Omega$ of $C_{2}$ contains the points of the grid $\Omega_{10}^{0}$. The inner points of $\Omega$ corresponds to the grid $\Omega_{6}^{1}$, the points not lying on the dashed boundary corresponds to the grid $\Omega_{8}^{s}$ and finally, the points not lying on the dot-and-dashed boundary corresponds to the grid $\Omega_{8}^{l}$. The numbers $1,2,4$ are the values of $\widetilde{\varepsilon}(y)$, the inner dots have $\widetilde{\varepsilon}(y)=8$.

the numbers of points in grids $\Omega_{M}^{t}, t \in\{0,1, s, l\}$ are the same. Fixing the basis $x=b_{1} \omega_{1}^{\vee}+b_{2} \omega_{2}^{\vee}$ results in the polynomial variables expressed as

$$
\begin{aligned}
& y_{1}=2\left(\cos \pi\left(2 b_{1}+b_{2}\right)+\cos \pi b_{2}\right), \\
& y_{2}=2\left(\cos 2 \pi\left(b_{1}+b_{2}\right)+\cos 2 \pi b_{1}\right) .
\end{aligned}
$$

Formula (8) specializes into

$$
I_{M}^{t}(f)=\frac{\pi^{2}}{4\left(M+h^{t}\right)^{2}} \sum_{y \in \Omega_{M}^{t}} \widetilde{\varepsilon}(y) K(y) f(y),
$$

where:

- $M \in \mathbb{N}$ is arbitrary;

- $h^{0}=1, h^{1}=4$ and $h^{s}=h^{l}=2$;

- the integration region $\Omega$, depicted in Fig. 4 , is bounded by two lines $y_{2}= \pm y_{1}-4$ and the parabola $y_{2}=\frac{y_{1}^{2}}{4}$

- the finite grid $\Omega_{M}^{t}$, depicted for $M=10$ in Fig. 4), consists of points $\left(y_{1}(x), y_{2}(x)\right)$ where $x=\frac{s_{1}^{t}}{M+h^{t}} \omega_{1}^{\vee}+\frac{s_{2}^{t}}{M+h^{t}} \omega_{2}^{\vee}$ with $s_{i}^{t}$ satisfying

$$
\begin{array}{ll}
s_{i}^{0} \in \mathbb{Z}^{\geq 0}, & 2 s_{1}^{0}+s_{2}^{0} \leq M, \\
s_{i}^{1} \in \mathbb{Z}^{>0}, & 2 s_{1}^{1}+s_{2}^{1}<M+4, \\
s_{1}^{s} \in \mathbb{Z}^{\geq 0}, s_{2}^{s} \in \mathbb{Z}^{>0}, & 2 s_{1}^{s}+s_{2}^{s} \leq M+2, \\
s_{1}^{l} \in \mathbb{Z}^{>0}, s_{2}^{l} \in \mathbb{Z}^{\geq 0}, & 2 s_{1}^{l}+s_{2}^{l}<M+2 ;
\end{array}
$$

- the weight function $K$ becomes

$$
K\left(y_{1}, y_{2}\right)=\sqrt{\left(y_{1}^{2}-4 y_{2}\right)\left(\left(y_{2}+4\right)^{2}-4 y_{1}^{2}\right)} ;
$$

- the weight function $\widetilde{\varepsilon}$ is equal to

$$
\widetilde{\varepsilon}(y)= \begin{cases}1 & \text { if }\left(y_{1}, y_{2}\right)=( \pm 4,4) \\ 2 & \text { if }\left(y_{1}, y_{2}\right)=(0,-4), \\ 8 & \text { if }\left(y_{1}, y_{2}\right) \text { is an inner point of } \Omega, \\ 4 & \text { otherwise }\end{cases}
$$



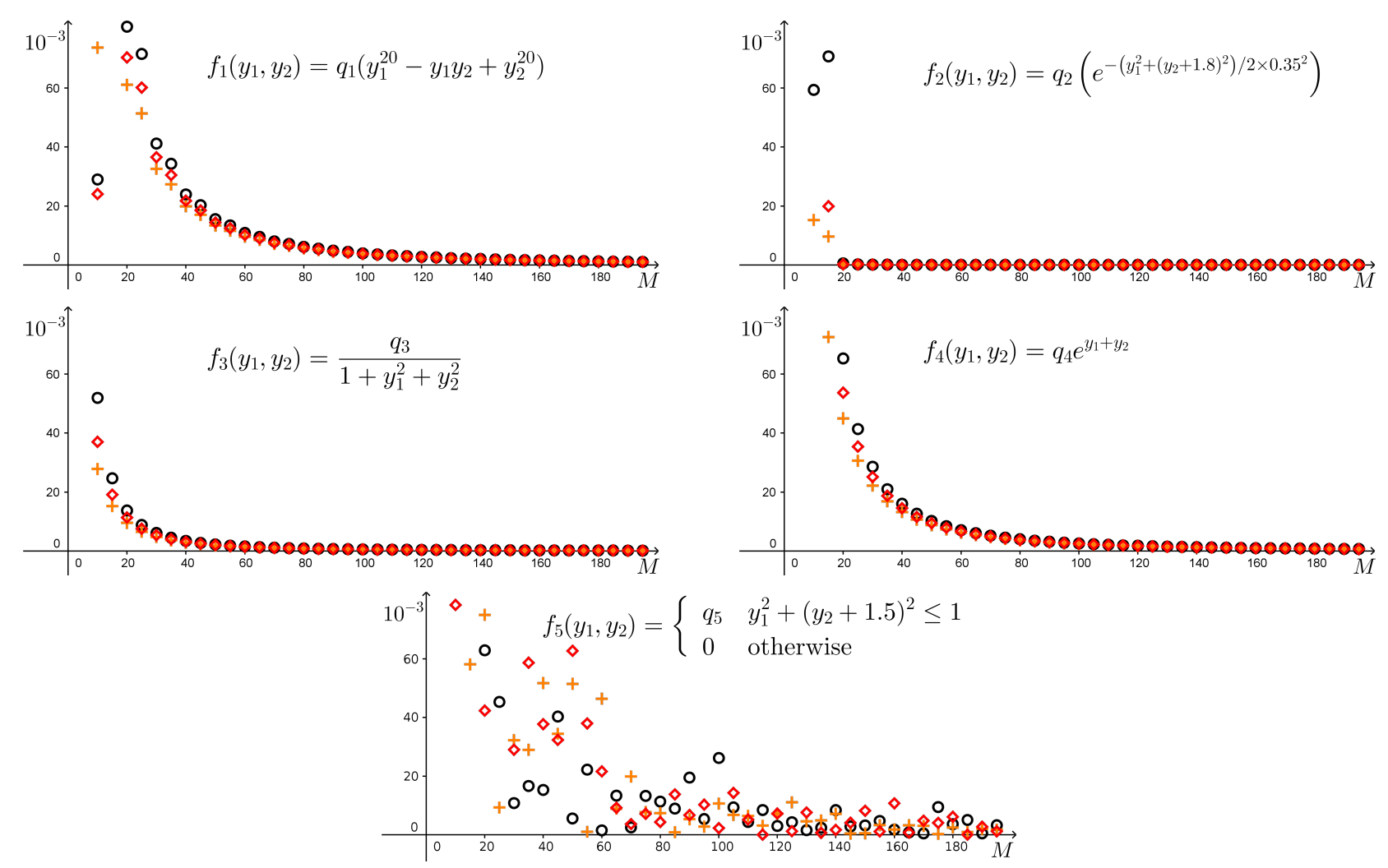

FiguRE 5. The graphs of error values $\left|1-I_{M}^{t}\left(f_{i}\right)\right|$ of the integral $I\left(f_{i}\right)=1$ and its estimations $I_{M}^{t}\left(f_{i}\right), M=$ $10,15,20, \ldots, 195$ given by (8). The values for $t=0,1, s$ are depicted as circles, "+" signs and diamonds, respectively.

For the purpose of numerical tests and comparison, we choose

$$
\begin{aligned}
& f_{1}\left(y_{1}, y_{2}\right)=q_{1}\left(y_{1}^{20}-y_{1} y_{2}+y_{2}^{20}\right), \\
& f_{2}\left(y_{1}, y_{2}\right)=q_{2}\left(e^{-\left(y_{1}^{2}+\left(y_{2}+1.8\right)^{2}\right) / 2 \times 0.35^{2}}\right), \\
& f_{3}\left(y_{1}, y_{2}\right)=\frac{q_{3}}{1+y_{1}^{2}+y_{2}^{2}}, \\
& f_{4}\left(y_{1}, y_{2}\right)=q_{4} e^{y_{1}+y_{2}}, \\
& f_{5}\left(y_{1}, y_{2}\right)= \begin{cases}q_{5} & \text { if } y_{1}^{2}+\left(y_{2}+1.5\right)^{2} \leq 1, \\
0 & \text { otherwise. }\end{cases}
\end{aligned}
$$

as model functions. Each value of $q_{i} \in \mathbb{R}$ is set to satisfy the condition $I\left(f_{i}\right)=1$. Fig. 5 shows for $M=10,15,20, \ldots, 195$ and $t \in\{0,1, s\}$ the graphs of the absolute value of the difference $\left|1-I_{M}^{t}\left(f_{i}\right)\right|$. Note that the cases $t=s$ and $t=l$ give the same results since $h^{s}=h^{l}=2$ and $K(y) f_{i}(y)$ vanish on the boundary of $\Omega$.

\section{Clenshaw-Curtis cubature FORMULAS}

\subsection{Clenshaw-Curtis method}

Assuming that we have an interpolation of a function $f$ in terms of $P\left(\lambda, k^{t}\right) \in \Pi_{M}$ in points $\Omega_{M}^{t}$, i.e.

$$
f \approx \sum_{\substack{\lambda \in P^{+} \\\langle\lambda, \eta\rangle \leq M}} b_{\lambda}^{t} P\left(\lambda, k^{t}\right)
$$

$$
f(y)=\sum_{\substack{\lambda \in P^{+} \\\langle\lambda, \eta\rangle \leq M}} b_{\lambda}^{t} P\left(\lambda, k^{t} ; y\right), \quad y \in \Omega_{M}^{t},
$$

we estimate a weighted integral of $f$ with a weight function $w$ over a domain $D \subset \Omega$ by

$$
\sum_{\substack{\lambda \in P^{+} \\\langle\lambda, \eta\rangle \leq M}} b_{\lambda}^{t} \int_{D} P\left(\lambda, k^{t} ; y\right) w(y) d y .
$$

Such construction of the Clenshaw-Curtis cubature rule implies the exact equality for any polynomial $f$ of $m$-degree at most $M$. Denoting

$$
a_{\lambda}^{t}(w) \equiv \int_{D} P\left(\lambda, k^{t} ; y\right) w(y) d y,
$$

the Clenshaw-Curtis cubature is thus given by

$$
\int_{D} f(y) w^{t}(y) d y \approx \sum_{\substack{\lambda \in P^{+} \\\langle\lambda, \eta\rangle \leq M}} b_{\lambda}^{t} a_{\lambda}^{t}(w),
$$

where the coefficients $b_{\lambda}^{t}$ and $a_{\lambda}^{t}(w)$ need to be determined. The coefficients $b_{\lambda}^{t}$ are readily obtained using the discrete orthogonality relations of the orbit functions from [13, 14. Denoting the order of the stabilizer of $\frac{\lambda+\varrho^{t}}{M+h^{t}}$ with respect to the dual affine Weyl group by $h_{\lambda+\varrho^{t}}^{\mathrm{V}}$, it holds that

$$
\begin{aligned}
& b_{\lambda}^{t}=\frac{\left|\operatorname{Stab}_{W}\left(\lambda+\varrho^{t}\right)\right|^{2}}{c|W|\left(M+h^{t}\right)^{n} h_{\lambda+\varrho^{t}}^{\vee}} \\
& \times \sum_{y \in \Omega_{M}^{t}} \widetilde{\varepsilon}(y) s^{t}(y) f(y) \overline{P\left(\lambda, k^{t} ; y\right)} .
\end{aligned}
$$




\begin{tabular}{|c|c|}
\hline \multicolumn{2}{|r|}{$A_{\left(\lambda_{1}, \lambda_{2}\right)}^{0}$} \\
\hline$\left(\lambda_{1}, \lambda_{2}\right)=(2 i, 2 j)$ & $\sum_{\left(\mu_{1}, \mu_{2}\right) \in \mathcal{M}(2 i, 2 j)} \frac{64\left(4 \mu_{2}^{2}+4 \mu_{1} \mu_{2}-3\right)}{\left|\operatorname{Stab}_{W}(2 i, 2 j)\right|\left(\mu_{1}^{2}-1\right)\left(4 \mu_{2}^{2}-1\right)\left(4 \mu_{2}^{2}-9\right)}$ \\
\hline$\left(\lambda_{1}, \lambda_{2}\right)=(2 i, 2 j+1)$ & $\sum_{\left(\mu_{1}, \mu_{2}\right) \in \mathcal{M}(2 i, 2 j+1)} \frac{-64\left(4 \mu_{2}^{2}+4 \mu_{1} \mu_{2}+3\right)}{\left|\operatorname{Stab}_{W}(2 i, 2 j+1)\right|\left(\mu_{1}^{2}-4\right)\left(4 \mu_{2}^{2}-1\right)\left(4 \mu_{2}^{2}-9\right)}$ \\
\hline Otherwise & 0 \\
\hline \multicolumn{2}{|r|}{$A_{\left(\lambda_{1}, \lambda_{2}\right)}^{1}$} \\
\hline $\begin{array}{l}\left(\lambda_{1}, \lambda_{2}\right)=(2 i, 2 j) \\
\left(\lambda_{1}, \lambda_{2}\right)=(2 i, 2 j+1) \\
\text { Otherwise }\end{array}$ & $\begin{array}{l}\frac{32(i+j+1)}{(2 i+4 j+3)(2 j+1)(2 i+1)} \\
\frac{32(j+1)}{(2 i+4 j+5)(2 i+2 j+3)(2 i+1)} \\
0\end{array}$ \\
\hline \multicolumn{2}{|r|}{$A_{\left(\lambda_{1}, \lambda_{2}\right)}^{s}$} \\
\hline$\left(\lambda_{1}, \lambda_{2}\right)=(2 i, 2 j)$ & $\sum_{\left(\mu_{1}, \mu_{2}\right) \in \mathcal{M}_{1}^{s}(2 i+1,2 j)} \frac{8\left(\mu_{1}+\mu_{2}\right)}{\left|\operatorname{Stab}_{W}(2 i+1,2 j)\right| \mu_{2}\left(\mu_{1}^{2}-1\right)\left(\mu_{2}^{2}-1\right)}$ \\
\hline$\left(\lambda_{1}, \lambda_{2}\right)=(2 i, 2 j+1)$ & $\sum_{\left(\mu_{1}, \mu_{2}\right) \in \mathcal{M}_{2}^{s}(2 i+1,2 j+1)} \frac{-8\left(\mu_{1}+\mu_{2}\right)}{\mu_{2}\left(\mu_{1}^{2}-1\right)\left(\mu_{2}^{2}-1\right)}$ \\
\hline Otherwise & 每 1 \\
\hline \multicolumn{2}{|r|}{$A_{\left(\lambda_{1}, \lambda_{2}\right)}^{l}$} \\
\hline$\left(\lambda_{1}, \lambda_{2}\right)=(2 i, 2 j)$ & $\left(\sum_{\left(\mu_{1}, \mu_{2}\right) \in \mathcal{M}_{1}^{l}(2 i, 2 j+1)}-\sum_{\left(\mu_{1}, \mu_{2}\right) \in \mathcal{M}_{2}^{l}(2 i, 2 j+1)}\right) \frac{32 \mu_{2}}{\left|\operatorname{Stab}_{W}(2 i, 2 j+1)\right| \mu_{1}\left(4 \mu_{2}^{2}-1\right)}$ \\
\hline$\left(\lambda_{1}, \lambda_{2}\right)=(2 i, 2 j+1)$ & $\left(\sum_{\left(\mu_{1}, \mu_{2}\right) \in \mathcal{M}_{2}^{l}(2 i, 2 j+2)}-\sum_{\left(\mu_{1}, \mu_{2}\right) \in \mathcal{M}_{1}^{l}(2 i, 2 j+2)}\right) \frac{16\left(2 \mu_{1} \mu_{2}+1\right)}{\left|\operatorname{Stab}_{W}(2 i, 2 j+2)\right|\left(\mu_{1}^{2}-1\right)\left(4 \mu_{2}^{2}-1\right)}$ \\
\hline Otherwise & - \\
\hline $\mathcal{M}\left(\lambda_{1}, \lambda_{2}\right)$ & $\left\{\left(\lambda_{1}+\lambda_{2}, \frac{\lambda_{1}}{2}+\lambda_{2}\right),\left(\lambda_{2}, \frac{\lambda_{1}}{2}+\lambda_{2}\right),\left(\lambda_{1}+\lambda_{2}, \frac{\lambda_{1}}{2}\right),\left(\lambda_{2},-\frac{\lambda_{1}}{2}\right)\right\}$ \\
\hline $\begin{array}{l}\mathcal{M}_{1}^{s}\left(\lambda_{1}, \lambda_{2}\right) \\
\mathcal{M}_{2}^{s}\left(\lambda_{1}, \lambda_{2}\right)\end{array}$ & $\begin{array}{l}\left\{\left(\lambda_{2}, \frac{\lambda_{1}}{2}+\lambda_{2}\right),\left(\lambda_{2},-\frac{\lambda_{1}}{2}\right)\right\} \\
\left\{\left(\lambda_{1}+\lambda_{2}, \frac{\lambda_{1}}{2}+\lambda_{2}\right),\left(\lambda_{1}+\lambda_{2}, \frac{\lambda_{1}}{2}\right)\right\}\end{array}$ \\
\hline $\begin{array}{l}\mathcal{M}_{1}^{l}\left(\lambda_{1}, \lambda_{2}\right) \\
\mathcal{M}_{2}^{l}\left(\lambda_{1}, \lambda_{2}\right)\end{array}$ & $\begin{array}{l}\left\{\left(\lambda_{1}+\lambda_{2}, \frac{\lambda_{1}}{2}+\lambda_{2}\right),\left(\lambda_{2}, \frac{\lambda_{1}}{2}+\lambda_{2}\right)\right\} \\
\left\{\left(\lambda_{1}+\lambda_{2}, \frac{\lambda_{1}}{2}\right),\left(\lambda_{2},-\frac{\lambda_{1}}{2}\right)\right\}\end{array}$ \\
\hline
\end{tabular}

TABLE 2. Values of $A_{\lambda}^{t}$ 111 for $t \in\{0,1, s, l\}, \lambda=\lambda_{1} \omega_{1}+\lambda_{2} \omega_{2}$ and $i, j$ are non-negative integers.

\begin{tabular}{ccc}
\hline$\left[\lambda_{0}, \lambda_{1}, \lambda_{2}\right]$ & $\left|\operatorname{Stab}_{W}\left(\lambda+\varrho^{t}\right)\right|$ & $h_{\lambda+\varrho^{t}}^{\vee}$ \\
\hline$(\star, \star, \star)$ & 1 & 1 \\
$(0, \star, \star)$ & 1 & 2 \\
$(\star, 0, \star)$ & 2 & 2 \\
$(\star, \star, 0)$ & 2 & 2 \\
$(0,0, \star)$ & 2 & 4 \\
$(0, \star, 0)$ & 2 & 8 \\
$(\star, 0,0)$ & 8 & 8 \\
\hline
\end{tabular}

TABLE 1. The values of $\left|\operatorname{Stab}_{W}\left(\lambda+\varrho^{t}\right)\right|$ and $h_{\lambda+\varrho^{t}}^{\vee}$ of $C_{2}$, where $\lambda+\varrho^{t}=\lambda_{1} \omega_{1}+\lambda_{2} \omega_{2}$ and $\lambda_{0} \equiv M+h^{t}-\lambda_{1}-$ $2 \lambda_{2}$. Asterisks denote non-zero positive integers.

It remains to evaluate the integrals $a_{\lambda}^{t}(w)$ which depend on the chosen weight and the integration domain $D \subset \Omega$.

Since the Jacobi polynomials have several properties connected to the domain $\Omega$ (e.g. continuous and discrete orthogonality), we firstly take $D=\Omega$. The cubature rules with the choice $w=w^{t}$ coincide for any simple Lie algebra with the formulas $(7)$. The difference lies in the fact that Clenshaw-Curtis method guarantees the exact equality only for polynomials up to $m$-degree $M$.

\subsection{Integration DOMAin $\Omega$ OF $C_{2}$}

In this section, the Clenshaw-Curtis integration method is applied to the algebra $C_{2}$. The values of $\left|\operatorname{Stab}_{W}\left(\lambda+\varrho^{t}\right)\right|$ and $h_{\lambda+\varrho^{t}}^{\vee}$, needed in 10$]$, are tabulated in Tab. 1 .

Since the choice of $w=w^{t}$ gives standard cubature formulas, the next natural choice of the weight function is to set $w=1$. In this case are the coefficients $a_{\lambda}^{t}(1)$, denoted by $A_{\lambda}^{t}$, expressed as the following integrals:

$$
A_{\lambda}^{t}=2 \pi^{2} \begin{cases}\int_{F} C_{\lambda}(x) S_{\varrho^{1}}(x) d x & \text { if } t=0 \\ \int_{F} S_{\lambda+\varrho^{1}}(x) d x & \text { if } t=1 \\ \int_{F} S_{\lambda+\varrho^{s}}^{s}(x) S_{\varrho^{l}}^{l}(x) d x & \text { if } t=s \\ \int_{F} S_{\lambda+\varrho^{l}}^{l}(x) S_{\varrho^{s}}^{s}(x) d x & \text { if } t=l .\end{cases}
$$

The exact values of $A_{\lambda}^{t}$ are explicitly calculated in Tab. 2

\subsection{Triangular domain of $C_{2}$}

The next choice of the domain $D$, for which we derive the Clenshaw-Curtis cubature rules, is the triangle $T \subset \Omega$ depicted on Fig. 6 and given explicitly by

$$
T \equiv\left\{\left(y_{1}, y_{2}\right) \mid y_{2} \leq 0,-\frac{y_{2}}{2}-2 \leq y_{1} \leq \frac{y_{2}}{2}+2\right\} \text {. }
$$




\begin{tabular}{|c|c|}
\hline \multicolumn{2}{|r|}{$\mathcal{A}_{\left(\lambda_{1}, \lambda_{2}\right)}^{0}$} \\
\hline $\begin{array}{l}\left(\lambda_{1}, \lambda_{2}\right)=(0,0) \\
\left(\lambda_{1}, \lambda_{2}\right)=(2 i, 2 j+1) \\
\text { Otherwise }\end{array}$ & $\begin{array}{l}\frac{\pi^{2}}{4} \\
(-1)^{i+1} \frac{8}{\left|\operatorname{Stab}_{W}(2 i, 2 j+1)\right|(2 i+2 j+1)(2 j+1)} \\
0\end{array}$ \\
\hline \multicolumn{2}{|r|}{$\mathcal{A}_{\left(\lambda_{1}, \lambda_{2}\right)}^{1}$} \\
\hline $\begin{array}{l}\left(\lambda_{1}, \lambda_{2}\right)=(0,0) \\
\left(\lambda_{1}, \lambda_{2}\right)=(2 i, 2 j), i+j \neq 0 \\
\left(\lambda_{1}, \lambda_{2}\right)=(2 i, 2 j+1) \\
\text { Otherwise }\end{array}$ & $\begin{array}{l}2 \pi^{2}+\frac{128}{9} \\
(-1)^{i+1} \frac{128(i+j+1)}{(2 i+2 j+1)(2 j-1)(2 i+2 j+3)(2 j+3)} \\
(-1)^{i+1} \frac{128(j+1)}{(2 j+1)(2 i+2 j+1)(2 j+3)(2 i+2 j+5)} \\
0\end{array}$ \\
\hline \multicolumn{2}{|r|}{$\mathcal{A}_{\left(\lambda_{1}, \lambda_{2}\right)}^{s}$} \\
\hline $\begin{array}{l}\left(\lambda_{1}, \lambda_{2}\right)=(0,0) \\
\left(\lambda_{1}, \lambda_{2}\right)=(2 i, 2 j), i+j \neq 0 \\
\left(\lambda_{1}, \lambda_{2}\right)=(2 i, 2 j+1) \\
\text { Otherwise }\end{array}$ & $\begin{array}{l}\pi^{2}+8 \\
(-1)^{i+1} \frac{16}{\left|\operatorname{Stab}_{W}(2 i+1,2 j)\right|(2 j+1)(2 i+2 j+1)(2 j-1)} \\
(-1)^{i+1} \frac{16}{(2 j+1)(2 i+2 j+3)(2 i+2 j+1)} \\
0\end{array}$ \\
\hline \multicolumn{2}{|r|}{$\mathcal{A}_{\left(\lambda_{1}, \lambda_{2}\right)}^{l}$} \\
\hline $\begin{array}{l}\left(\lambda_{1}, \lambda_{2}\right)=(0,0) \\
\left(\lambda_{1}, \lambda_{2}\right)=(2 i, 2 j+1) \\
\text { Otherwise }\end{array}$ & $\begin{array}{l}\pi^{2} \\
(-1)^{i+1} \frac{128(i+j+1)(j+1)}{\left|\operatorname{Stab}_{W}(2 i, 2 j+2)\right|(2 i+2 j+3)(2 j+3)(2 i+2 j+1)(2 j+1)} \\
0\end{array}$ \\
\hline
\end{tabular}

TABle 3. Values of $\mathcal{A}_{\lambda}^{t}$, given by $(12)$, for $t \in\{0,1, s, l\}$ and $\lambda=\lambda_{1} \omega_{1}+\lambda_{2} \omega_{2}$. The indices $i, j$ are non-negative integers.

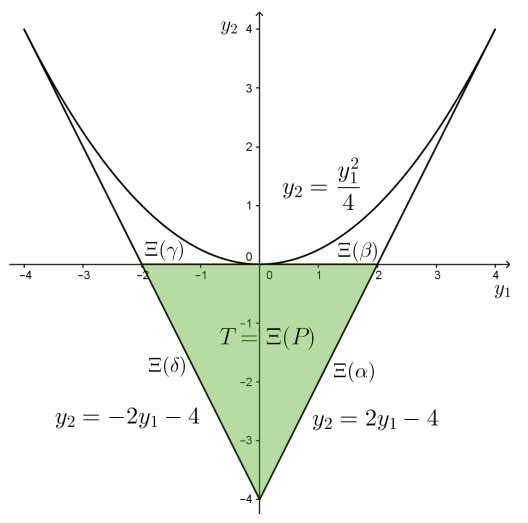

Figure 6 . The domain bounded by the two lines and the parabola with the inscribed triangle $T$ corresponds to the integration region $\Omega$ of $C_{2}$.

Choosing the weight function $w=w^{t}$, the integrals $a_{\lambda}^{t}\left(w^{t}\right)$ are calculated by a change of variables induced by the map $\Xi$. Denoting $\mathcal{A}_{\lambda}^{t} \equiv a_{\lambda}^{t}\left(w^{t}\right)$, it holds that

$$
\mathcal{A}_{\lambda}^{t}=2 \pi^{2} \int_{P} S_{\lambda+\varrho^{t}}^{t}(x) \overline{S_{\varrho^{t}}^{t}(x)} d x
$$

where $P$ is the pre-image of the triangle $T$ under the map $\Xi$. This pre-image $P$, depicted as a square in Fig. 7. contains the points $b_{1} \omega_{1}^{\vee}+b_{2} \omega_{2}^{\vee}$ satisfying $2 b_{1}+b_{2} \geq 1 / 2,2 b_{1}+b_{2} \leq 1, b_{2} \geq 0$ and $b_{2} \leq 1 / 2$. The exact values of $\mathcal{A}_{\lambda}^{t}$ are tabulated in Tab. 3

Finally, choosing $w=1$, we calculate the coefficients

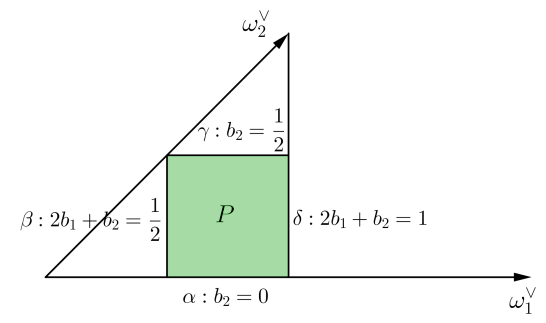

Figure 7. The fundamental domain $F$ corresponding to $C_{2}$ is depicted as the triangle containing the square $P$ with the boundaries $\alpha, \beta, \gamma$ and $\delta$.

$\mathcal{B}_{\lambda}^{t} \equiv a_{\lambda}^{t}(1)$ on $T$ as the following integrals

$$
\mathcal{B}_{\lambda}^{t}=2 \pi^{2} \begin{cases}\int_{P} C_{\lambda}(x) S_{\varrho^{1}}(x) d x & \text { if } t=0, \\ \int_{P} S_{\lambda+\varrho^{1}}(x) d x & \text { if } t=1, \\ \int_{P} S_{\lambda+\varrho^{s}}^{s}(x) S_{\varrho^{l}}^{l}(x) d x & \text { if } t=s, \\ \int_{P} S_{\lambda+\varrho^{l}}^{l}(x) S_{\varrho^{s}}^{s}(x) d x & \text { if } t=l .\end{cases}
$$

The exact values of $\mathcal{B}_{\lambda}^{t}$ are tabulated in Tab. 4

We choose the following functions as model functions for numerical tests,

$$
\begin{aligned}
& g_{1}\left(y_{1}, y_{2}\right)=r_{1}\left(y_{1}^{20}-y_{1} y_{2}+y_{2}^{20}\right), \\
& g_{2}\left(y_{1}, y_{2}\right)=r_{2}\left(e^{-\left(y_{1}^{2}+\left(y_{2}+1.8\right)^{2}\right) / 2 \times 0.35^{2}}\right), \\
& g_{3}\left(y_{1}, y_{2}\right)=\frac{r_{3}}{1+y_{1}^{2}+y_{2}^{2}}, \\
& g_{4}\left(y_{1}, y_{2}\right)=r_{4} e^{x+y}
\end{aligned}
$$




\begin{tabular}{|c|c|}
\hline & $\mathcal{B}_{\left(\lambda_{1}, \lambda_{2}\right)}^{0}$ \\
\hline$\left(\lambda_{1}, \lambda_{2}\right)=(0,1)$ & $\frac{-32}{3}$ \\
\hline$\left(\lambda_{1}, \lambda_{2}\right)=(2,0)$ & $\frac{-16}{3}$ \\
\hline$\left(\lambda_{1}, \lambda_{2}\right)=(2 i, 2), i \neq 0$ & $\frac{16\left(1+(-1)^{i+1}\right)}{3 i(i+1)}$ \\
\hline$\left(\lambda_{1}, \lambda_{2}\right)=(2 i, 1), i \neq 0$ & $16\left[\frac{2}{(2 i+3)(2 i-1)}+\frac{1+(2 i+1)(-1)^{i+1}}{3 i(i+1)}\right]$ \\
\hline$\left(\lambda_{1}, \lambda_{2}\right)=(2 i, 2 j+1), j \neq 0$ & $\frac{64}{\left|\operatorname{Stab}_{W}(2 i, 2 j+1)\right|}\left[\frac{-1+(2 j+1)(-1)^{j}}{\left((2 i+2 j+1)^{2}-4\right)\left((2 j+1)^{2}-1\right)}+\frac{-1+(2 i+2 j+1)(-1)^{i+j}}{\left((2 i+2 j+1)^{2}-1\right)\left((2 j+1)^{2}-4\right)}\right]$ \\
\hline$\left(\lambda_{1}, \lambda_{2}\right)=(2 i, 2 j), j \neq 1, i+j \neq 1$ & $\frac{16}{\left|\operatorname{Stab}_{W}(2 i, 2 j)\right|}\left[\frac{1+(-1)^{i+j}}{\left((i+j)^{2}-1\right)\left(4 j^{2}-1\right)}+\frac{1+(-1)^{j}}{\left(4(i+j)^{2}-1\right)\left(j^{2}-1\right)}\right]$ \\
\hline Otherwise & 0 \\
\hline & $\mathcal{B}_{\left(\lambda_{1}, \lambda_{2}\right)}^{1}$ \\
\hline$\left(\lambda_{1}, \lambda_{2}\right)=(2 i, 2 j)$ & $\frac{4\left(1+(-1)^{i+j}\right)}{(i+j+1)(2 j+1)}$ \\
\hline$\left(\lambda_{1}, \lambda_{2}\right)=(2 i, 2 j+1)$ & $\frac{-4\left(1+(-1)^{j}\right)}{(2 i+2 j+3)(j+1)}$ \\
\hline Otherwise & 0 \\
\hline & $\mathcal{B}_{\left(\lambda_{1}, \lambda_{2}\right)}^{s}$ \\
\hline$\left(\lambda_{1}, \lambda_{2}\right)=(0,0)$ & 8 \\
\hline$\left(\lambda_{1}, \lambda_{2}\right)=(2 i, 1)$ & $\frac{16}{(2 i+3)(2 i+1)}$ \\
\hline$\left(\lambda_{1}, \lambda_{2}\right)=(2 i, 2 j), i+j \neq 0$ & $\frac{8\left(1+(2 i+2 j+1)(-1)^{i+j+1}\right)}{\left|\operatorname{Stab}_{W}(2 i+1,2 j)\right|(i+j+1)(i+j)\left(4 j^{2}-1\right)}$ \\
\hline$\left(\lambda_{1}, \lambda_{2}\right)=(2 i, 2 j+1), j \neq 0$ & $\frac{8\left(-1+(2 j+1)(-1)^{j}\right)}{(2 i+2 j+3)(2 i+2 j+1) j(j+1)}$ \\
\hline Otherwise & 0 \\
\hline & $\mathcal{B}_{\left(\lambda_{1}, \lambda_{2}\right)}^{l}$ \\
\hline$\left(\lambda_{1}, \lambda_{2}\right)=(0,0)$ & 8 \\
\hline$\left(\lambda_{1}, \lambda_{2}\right)=(2 i, 0), i \neq 0$ & $8\left[\frac{2 i+1+(-1)^{i+1}}{2 i(i+1)}+\frac{1}{2 i+1}\right]$ \\
\hline$\left(\lambda_{1}, \lambda_{2}\right)=(2 i, 2 j), j \neq 0$ & $\frac{4}{\left|\operatorname{Stab}_{W}(2 i, 2 j+1)\right|}\left[\frac{2 i+2 j+1+(-1)^{i+j+1}}{(i+j+1)(2 j+1)(i+j)}+\frac{2 j+1+(-1)^{j+1}}{(2 i+2 j+1)(j+1) j}\right]$ \\
\hline$\left(\lambda_{1}, \lambda_{2}\right)=(2 i, 2 j+1)$ & $\frac{16}{\left|\operatorname{Stab}_{W}(2 i, 2 j+2)\right|}\left[\frac{\left(-1+(-1)^{j+1}\right)(i+j+1)}{(2 i+2 j+3)(j+1)(2 i+2 j+1)}+\frac{\left(-1+(-1)^{i+j+1}\right)(j+1)}{(i+j+1)(2 j+3)(2 j+1)}\right]$ \\
\hline Otherwise & 0 \\
\hline
\end{tabular}

TABLE 4. Values of $\mathcal{B}_{\lambda}^{t}$, given by (13), for $t \in\{0,1, s, l\}$ and $\lambda=\lambda_{1} \omega_{1}+\lambda_{2} \omega_{2}$. The indices $i, j$ are non-negative integers.

$$
g_{5}\left(y_{1}, y_{2}\right)= \begin{cases}r_{5} & \text { if } y_{1}^{2}+\left(y_{2}+1.5\right)^{2} \leq 1 \\ 0 & \text { otherwise }\end{cases}
$$

Each value of $r_{i} \in \mathbb{R}$ is set to satisfy the normalization condition $\int_{T} g_{i}(y) d y=1$. We compute the approximations

$$
\mathcal{I}_{M}^{t}\left(g_{i}\right)=\sum_{\substack{\lambda \in P^{+} \\\langle\lambda, \eta\rangle \leq M}} b_{\lambda}^{t} \mathcal{B}_{\lambda}^{t}
$$

of $\int_{T} g_{i}(y) d y=1$ with the formula for $\mathcal{B}_{\lambda}^{t}$ given by (13). Figs. 8 and 9 show for $t \in\{0,1, s, l\}$ the graphs of of the absolute value of the difference $\left|1-\mathcal{I}_{M}^{t}\left(g_{i}\right)\right|$.

\section{Concluding Remarks}

(1.) Establishing the explicit connection between the Jacobi and Macdonald polynomials and the Weyl group orbit functions in Section 2.3 forms a crucial step for generalizing known cubature formulas to the entire class of the Jacobi polynomials.

(2.) Numerical tests results in Figs. 5 and 8 indicate in general excellent convergence rates of the developed cubature rules including their Clenshaw-Curtis 

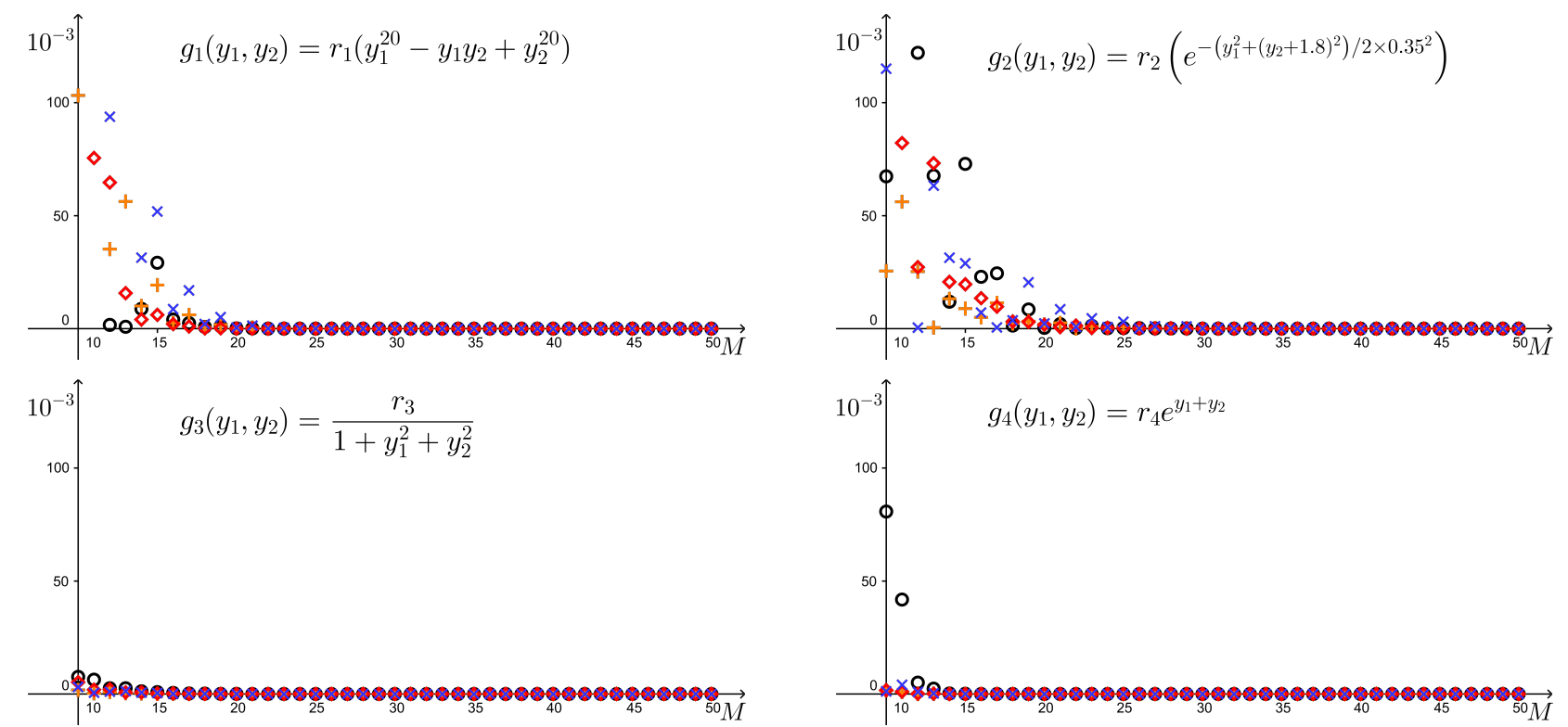

Figure 8. The graphs of error values $\left|1-\mathcal{I}_{M}^{t}\left(g_{i}\right)\right|$ of the integral $\int_{T} g_{i}(y) d y=1, i=1, \ldots, 4$ and its approximations $\mathcal{I}_{M}^{t}\left(g_{i}\right), M=10,11, \ldots, 50$ given by (14). The values for $t=0,1, s, l$ are depicted as circles, "+", diamonds and " $\times$ ", respectively.

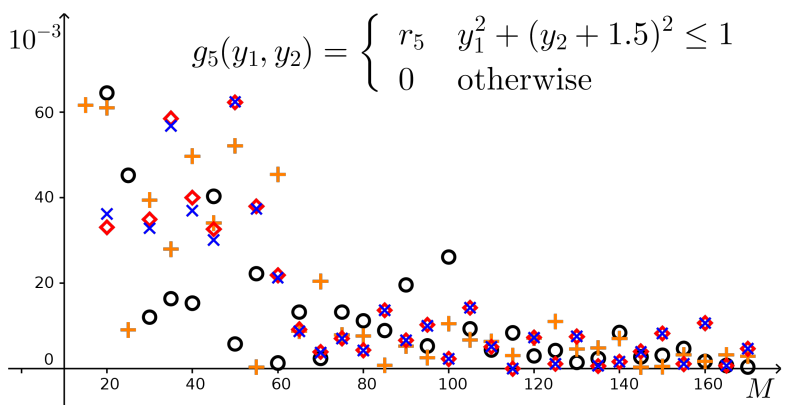

Figure 9. The graphs of error values $\left|1-\mathcal{I}_{M}^{t}\left(g_{5}\right)\right|$ of the integral $\int_{T} g_{5}(y) d y=1$ and its approximations $\mathcal{I}_{M}^{t}\left(g_{5}\right), M=10,15,20, \ldots, 170$ given by (14). The values for $t=0,1, s, l$ are depicted as circles, "+", diamonds and " $\times$ ", respectively.

versions for the case $C_{2}$. The convergence rate of the multidimensional step-functions, even though less uniform, still appears to be very good. Developing similar methods for the two-variable case $G_{2}$ and extending the rules to higher dimensions poses an open problem.

(3.) The hyperinterpolation methods [3, 25, 34, 35] are among the tools which directly use cubature rules. For the standard cubature rules of the Weyl group orbit functions, several tests with very good results are also performed in [15]. Developing and testing hyperinterpolation methods for the presented cubature rules merits further study.

(4.) The present work demonstrates wide variety of possibilities of constructing the cubature rules in the orbit functions setting. Comparison of the developed methods is necessary for establishing range of their viable applications. Especially, comparison of the Gauss and Clenshaw-Curtis cubature methods, similar to [37, regarding their efficiency, speed, model function and integration domain dependence merits further research.

\section{ACKNOWLEDGMEnts}

LM and JH gratefully acknowledge the support of this work by RVO68407700.

\section{REFERENCES}

[1] H. Berens, H. J. Schmid, Y. Xu, Multivariate Gaussian cubature formulae, Arch. Math. (Basel) 64 (1995), no. 1, 26-32, DOI:10.1007/BF01193547.

[2] N. Bourbaki, Groupes et algèbres de Lie, Chapitres IV, V, VI, Hermann, Paris, 1968.

[3] M. Caliari, S. De Marchi, M. Vianello, Hyperinterpolation in the cube, Comput. \& Math. with Appl. 55 (2008), 2490-2497, DOI:10.1016/j.camwa.2007.10.003

[4] D. Chernyshenko, H. Fangohr, Computing the demagnetizing tensor for finite difference micromagnetic simulations via numerical integration, J. Magn. Magn. Mat. 381 (2015) 440-445, DOI:10.1016/j.jmmm.2015.01.013

[5] C.W. Clenshaw, A.R. Curtis, A method for numerical integration on an automatic computer, Numer. Math. 2 (1960), 197-205, DOI:10.1007/BF01386223.

[6] R. Cools, An encyclopaedia of cubature formulas, Journal of Complexity 19 (2003), 445-453, DOI:10.1016/S0885-064X(03)00011-6

[7] R. Cools, I. P. Mysovskikh, H. J. Schmid, Cubature formulae and orthogonal polynomials, J. Comput. Appl. Math. 127 (2001), no. 1-2, 121-152, DOI:10.1016/S0377-0427(00)00495-7 
[8] P. de la Harpe, C. Pache, B. Venkov, Construction of spherical cubature formulas using lattices, Algebra i Analiz 18 (2006), no. 1, 162-186; reprinted in St. Petersburg Math. J. 18 (2007), no. 1, 119-139, DOI:10.1090/S1061-0022-07-00946-6

[9] D. C. Handscomb, J. C. Mason, Chebyshev polynomials, Chapman\&Hall/CRC, USA, 2003, DOI:10.1201/9781420036114

[10] L. Háková, J. Hrivnák, J. Patera, Four families of Weyl group orbit functions of $B_{3}$ and $C_{3}$, J. Math. Phys. 54 (2013), 083501, 19, DOI:10.1063/1.4817340

[11] G. Heckman, H. Schlichtkrull, Harmonic Analysis and Special Functions on Symmetric Spaces, Academic Press Inc., San Diego, 1994.

[12] G. Heckman, E. M. Opdam, Root systems and hypergeometric functions. I, II, Composition Math. 64 (1987), 329-373.

[13] J. Hrivnák, J. Patera, On discretization of tori of compact simple Lie groups, J. Phys. A: Math. Theor. 42 (2009) 385208, DOI:10.1088/1751-8113/42/38/385208

[14] J. Hrivnák, L. Motlochová, J. Patera, On discretization of tori of compact simple Lie groups II., J. Phys. A 45 (2012), 255201, 18, DOI:10.1088/1751-8113/45/25/255201

[15] J. Hrivnák, L. Motlochová, J. Patera, Cubature formulas of multivariate polynomials arising from symmetric orbit functions, arXiv:1512.01710.

[16] J. E. Humphreys, Introduction to Lie algebras and representation theory, Spinger-Verlag, New York, 1978, DOI:10.1007/978-1-4612-6398-2

[17] A. Klimyk, J. Patera, (Anti)symmetric multivariate trigonometric functions and corresponding Fourier transforms, J. Math. Phys. 48 (2007), 093504, 24, DOI:10.1063/1.2779768

[18] A. U. Klimyk, J. Patera, Orbit functions, SIGMA 2 (2006), 006, 60 pages, DOI:10.3842/SIGMA.2006.006

[19] A. U. Klimyk, J. Patera, Antisymmetric orbit functions, SIGMA 3 (2007), paper 023, 83 pages, DOI:10.3842/SIGMA.2007.023

[20] T. H. Koornwinder, Two-variable analogues of the classical orthogonal polynomials, Theory and Application of Special Functions, edited by R. A. Askey, Academic Press, New York (1975) 435-495, DOI:10.1016/B978-0-12-064850-4.50015-X

[21] H. Li, J. Sun, Y. Xu, Discrete Fourier Analysis and Chebyshev Polynomials with $G_{2}$ Group, SIGMA 8, Paper 067, 29, 2012, DOI:10.3842/SIGMA.2012.067

[22] H. Li, J. Sun, Y. Xu, Discrete Fourier analysis, cubature and interpolation on a hexagon and a triangle, SIAM J. Numer. Anal. 46 (2008), 1653-1681, DOI: $10.1137 / 060671851$

[23] H. Li, Y. Xu, Discrete Fourier analysis on fundamental domain and simplex of $A_{d}$ lattice in d-variables, J. Fourier Anal. Appl. 16, 383-433, (2010), DOI:10.1007/s00041-009-9106-9.

[24] I. G. Macdonald, Orthogonal polynomials associated with root systems, Sém. Lothar. Combin. 45 (2000/01), Art. B45a, 40, DOI:10.1007/978-94-009-0501-6_14.
[25] S. De Marchi, M. Vianello, Y. Xu, New cubature formulae and hyperinterpolation in three variables, BIT. Numerical Mathematics 49 (2009), Number 1, 55-73, DOI:10.1007/s10543-009-0210-7.

[26] R. V. Moody, L. Motlochová, J. Patera, Gaussian cubature arising from hybrid characters of simple Lie groups , J. Fourier Anal. Appl. 20 (2014), Issue 6, 1257-1290, DOI:10.1007/s00041-014-9355-0

[27] R. V. Moody, J. Patera, Cubature formulae for orthogonal polynomials in terms of elements of finite order of compact simple Lie groups, Adv. in Appl. Math. 47 (2011) 509-535, DOI:10.1016/j.aam.2010.11.005

[28] R. V. Moody and J. Patera, Orthogonality within the families of $C$-, $S$-, and E-functions of any compact semisimple Lie group, SIGMA 2 (2006) 076, 14 pages, DOI:10.3842/SIGMA.2006.076.

[29] L. Motlochová, Special functions of Weyl groups and their continuous and discrete orthogonality, Ph.D. Thesis, Université de Montréal (2014), http://hdl.handle.net/1866/11153

[30] H. Z. Munthe-Kaas, M. Nome, B. N. Ryland, Through the kaleidoscope: symmetries, groups and Chebyshevapproximations from a computational point of view, Foundations of computational mathematics, Budapest 2011, 188-229, London Math. Soc. Lecture Note Ser., 403, Cambridge Univ. Press, Cambridge, 2013.

[31] B. N. Ryland, H. Z. Munthe-Kaas, On multivariate Chebyshev polynomials and spectral approximation on triangles, Spectral and High Order Methods for Partial Differential Equations, Lecture Notes in computational science and engineering, Springer, 2011, DOI:10.1007/978-3-642-15337-2_2

[32] H. J. Schmid, Y. Xu, On bivariate Gaussian cubature formulae, Proc. Amer. Math. Soc., 122 (1994), 833-841, DOI: $10.2307 / 2160762$

[33] I. Sfevanovic, F. Merli, P. Crespo-Valero, W. Simon, S. Holzwarth, M. Mattes, J. R. Mosig, Integral Equation Modeling of Waveguide-Fed Planar Antennas, IEEE

Antenn. Propag. M. 51 (2009), 82-92, DOI:10.1109/MAP.2009.5433099

[34] I. H. Sloan, Polynomial interpolation and hyperinterpolation over general regions, J. Approx. Theory 83 (1995), no. 2, 238-254, DOI:10.1006/jath.1995.1119.

[35] A. Sommariva, M. Vianello, R. Zanovello, Nontensorial Clenshaw-Curtis cubature, Numer. Algorithms 49 (2008), Number 1-4, 409-427, DOI:10.1007/s11075-008-9203-x.

[36] G. Szegö, Orthogonal polynomials, American Mathematical Society, Providence, R.I., 1975.

[37] L. N. Trefethen, Is Gauss quadrature better than Clenshaw-Curtis? SIAM Rev. 50 (2008) 67-87, DOI:10.1137/060659831

[38] J. Waldvogel, Fast construction of the Fejér and Clenshaw-Curtis quadrature rules, BIT 46 (2006), no. 1, 195-202, DOI:10.1007/s10543-006-0045-4.

[39] J. C. Young, S. D. Gedney, R. J. Adams, QuasiMixed-Order Prism Basis Functions for Nyström-Based Volume Integral Equations, IEEE Trans. Magn. 48 (2012), 2560-2566, DOI:10.1109/TMAG.2012.2197634. 